\title{
Methodology for Cage Shape Optimization of a Permanent Magnet Synchronous Motor Under Line Start Conditions
}

\author{
Cezary Jędryczka $^{\circledR 1}$, Łukasz Knypiński ${ }^{\circledR 1}$, Andrzej Demenko ${ }^{1}$, and Jan K. Sykulski ${ }^{\circledR}$, Fellow, IEEE \\ ${ }^{1}$ Institute of Electrical Engineering and Electronics, Poznan University of Technology, 60-965 Poznań, Poland \\ ${ }^{2}$ Electronics and Computer Science, University of Southampton, Southampton SO17 1BJ, U.K.
}

\begin{abstract}
This paper proposes a methodology for shape optimization of the starting cage of a line start permanent magnet synchronous motor motor with the aim to improve its synchronization performance. The parameters of the machine are established from a field-circuit model, where the magnetic field is simulated using a finite element method (FEM). A strategy for evaluating machine parameters exploiting parallel computing is proposed. To facilitate the use of FEM package, bespoke procedures have been developed and model parameterization applied with the aid of the scripting language Visual Basic. A particle swarm algorithm has been adapted for design optimization purposes. The proposed strategy has been verified via test simulations.
\end{abstract}

Index Terms-Electromagnetic field, finite-element analysis, line start permanent magnet synchronous motor, optimization methods.

\section{INTRODUCTION}

$\mathbf{T}$ HE global drive for energy efficiency inspired the search for new types of permanent magnet synchronous motors with the ability for line start. The development of such motors depends on the availability of reliable methods for analysis, design, and optimization. A particular challenge is to find a cage shape allowing synchronization even under high inertia load. With the aim to find a reliable methodology, in this paper an accurate electromagnetic model has been derived in combination with a computationally efficient algorithm for solving the resultant equations. The field-circuit model encompasses: 1) electromagnetic field equations taking into account the skin effect in the cage bars; 2) a description of the driving circuit including winding connection; and 3) mechanical formulation, where the electromagnetic torque is expressed in terms of field quantities.

The parameters resulting in the best starting performance of the line start permanent magnet synchronous motor motor (LSPMSM) are normally different to the optimal design for synchronous operation (efficiency and power factor) [1], [2], thus it is advisable to conduct a multiobjective search to account for all conflicting requirements. This type of optimization is very time consuming if full numerical modeling is used; in this paper, efficient design strategies are considered as well as ways of extracting motor parameters from field solutions. An algorithm is developed to establishvia parallel computation-such parameters, primarily the synchronous torque and the synchronizing torque during starting. The methodology first proposed in [2] has been adapted relying on numerical field solutions using finite-element modeling at an imposed speed.

Various optimization methods were considered, including an approach described in [3]. Following many tests and

Manuscript received June 27, 2017; revised August 29, 2017 and September 25, 2017; accepted October 13, 2017. Corresponding author: C. Jędryczka (e-mail: cezary.jedryczka@put.poznan.pl).

Color versions of one or more of the figures in this paper are available online at http://ieeexplore.ieee.org.

Digital Object Identifier 10.1109/TMAG.2017.2764680 practical experience with numerical analysis, it was decided that methods exploiting particle swarm optimization (PSO) were most appropriate for the efficient design of the LSPMSM.

\section{Modeling Strategy And Techniques}

In the design of LSPMSMs, various constraints must be met and specific requirements satisfied regarding both steadystate synchronous operation and asynchronous starting performance. Lumped parameter models are typically used for analysis [2]. Unfortunately, such models tend to be unreliable, especially in the context of the transient state during starting; hence, their usefulness is very limited for design optimization purposes. This limitation provided motivation to search for more accurate approaches based on field models. Originally a sequential algorithm was applied, where a steady state was reached via a simulation of a transient start-up phase. In this algorithm, first the start-up and then the steady-state parameters were established. Calculations were performed for a given value of the load inertia $J_{L}$, specified with the help of a coefficient $k_{i}$ defined by the ratio of the moment of inertia of the load system to that of the rotor of the motor itself. To assess the starting parameters of the motor, the maximum value $k_{\text {imax }}$ is used, above which the motor fails to synchronize. In order to establish this maximum value, it is necessary to repeat simulations with increasing values of $k_{i}$. Finding the value of $k_{\text {imax }}$ by these repetitive simulations of the motor startup results in unacceptably long computing times, in particular as the number of necessary function calls is often unknown in advance. Such sequential strategy may therefore be inefficient.

In the search for a computationally efficient approach, it was therefore proposed to implement a parallel methodology where the transient start-up and steady-state simulations are performed simultaneously as two independent computational processes. The relevant start-up parameters are assessed using the approach described in [4], where the parameters are evaluated based on the value of the synchronizing torque $T_{80}$ defined as the asynchronous torque generated by the cage winding at a speed close to the final operating speed 
a)

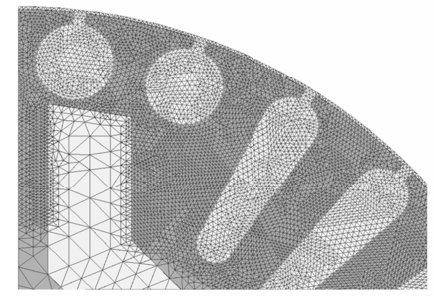

b)

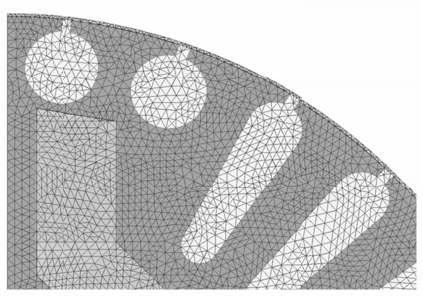

Fig. 1. Finite-element mesh. (a) For starting. (b) For steady-state operation.

(here taken as $80 \%$ of the synchronous speed). The value of this torque may be found using finite-element modeling at a prescribed speed, i.e., neglecting the mechanical transient, thus significantly reducing the computational effort required to establish the component of the objective function representing the synchronizing parameters of the machine.

Two possible strategies were considered: 1) based on two discretization meshes for the two states (asynchronous and synchronous) and 2) using identical meshes. Strategy 1) bears a resemblance to an adaptive mesh refinement approach. The mesh of the stator is the same for both modes of operation, but the rotor area is discretized differently with a refined mesh in the air gap region of the slot pitch (see Fig. 1).

Comparisons were made between the calculated start-up parameters using the mesh optimized for the steady-state simulations and values obtained from the mesh adapted so as to better capture the transient performance during the startup period at $80 \%$ of the synchronous speed. The differences were noticeable, reaching a few percent of the synchronizing torque $T_{80}$. Moreover, it was observed that using an adapted mesh allowed for a reduction of computing times up to about $15 \%$, depending on the level of magnetic saturation and related number of iterations in the Newton-Raphson algorithm.

In the model dedicated to the steady-state operation [synchronous mode (SM)] at synchronous speed, the dynamics of the moving elements was considered assuming a relatively small value of the load moment of inertia $\left(k_{i}=1\right)$ and the rotor speed at the instant of switching ON equal to the speed of the rotating field. This has allowed shortening of the simulated transient associated with the switching ON of the motor. A criterion was used for two simulation cycles not to differ by more than $1 \%$ in terms of efficiency and the power factor for the steady state to be assumed to have been reached. A similar strategy was applied to the simulation of the asynchronous state [asynchronous mode (ASM)] with imposed speed. For both types of simulation (SM and ASM), a 2-D formulation was used in terms of magnetic vector potential and time stepping with the same time step $\Delta t$.

It should be noted that in order to accomplish the desired parallelization - and achieve the automation of the execution of the relevant tasks-dedicated scripts had to be developed associating the algorithms for field modeling at start-up and steady state. Moreover, both algorithms have been linked to the optimization routines explained later in this paper. The overall concept of the design optimization of the LSPMSM is explained in Fig. 2. The calculations presented here have been performed for a thermally steady-state condition, i.e., constant properties of permanent magnets and electric conductivities.

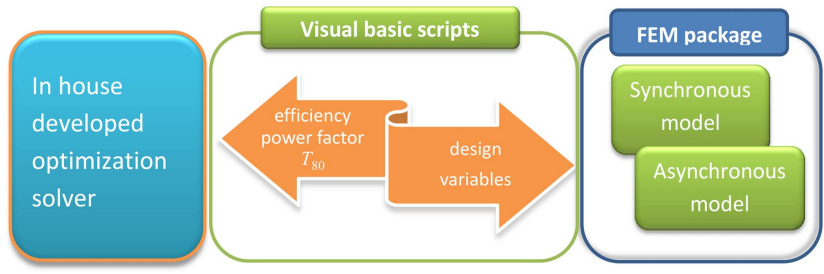

Fig. 2. Proposed concept of design optimization of LSPMSM.

The inclusion of the end-ring effects is essential when using 2-D finite element method (FEM) models and the results should be verified either by full 3-D analysis or by measurements on a real machine. The 2-D FEM models developed for the study presented in this paper had been originally validated by measurements on a prototype of a motor with round bars [5], and the method of estimating the end-ring lumped parameters was then extended to include the dropshaped bars.

\section{Optimization Algorithm}

Despite the implementation of the efficient strategy for code parallelization, the optimization process remains computationally time consuming due to the need for repetitive simulations with the continuously varied cage parameters; hence the need for an effective optimization algorithm. Many alternatives were considered, including genetic [3], particle swarm [6] and ant colony algorithms [7], as well as the less popular brain storm [8], bat-inspired [9] and gray wolf optimizers [10]. Following intensive search and testing, it was decided to rely on the well performing particle swarm approach, with a modification of an added coefficient representing the swarm center.

The PSO algorithm, inspired by the flocking and schooling patterns of birds and fish, was first introduced in 1995 [11]. In the PSO, the swarm consists of particles. In the optimization process, the particles move in the $n$-dimensional design space, where $n$ refers to the number of design parameters. Each $i$ th particle in the swarm is described by its position $x^{i}$ and velocity $v^{i}$ representing the possible variant of the machine. Each particle remembers its best position in previous time steps $\boldsymbol{x}_{L}^{i}$ and the position of the leader $\boldsymbol{x}_{G}$, which is the fittest particle in the swarm. In the classical PSO method in the $k$ th time step, the position vector of the particle is derived from the following formula:

$$
\boldsymbol{x}_{k}^{i}=\boldsymbol{x}_{k-1}^{i}+w \boldsymbol{v}_{k-1}^{i}+c_{1} r_{1}\left(\boldsymbol{x}_{L}^{i}-\boldsymbol{x}_{k-1}^{i}\right)+c_{2} r_{2}\left(\boldsymbol{x}_{G}-\boldsymbol{x}_{k-1}^{i}\right)
$$

where $w$ is the inertia factor, $\boldsymbol{x}_{k-1}^{i}$ is the position vector in the previous step $k-1 ; c_{1}$ and $c_{2}$ are the learning coefficients, and $r_{1}$ and $r_{2}$ are the random numbers from the range $(0,1)[12]$.

Since the original introduction of the classical PSO, this population-based stochastic optimization approach has been successfully applied to many engineering problems. The more advanced applications include modifications aiming at improving the convergence and accuracy [13]-[15].

In this paper, a modified version has been utilized, adapted to particular requirements of the design of electromechanical 
devices. In the proposed algorithm, a term is added to (1) which describes the center of gravity of the swarm

$$
c_{3} r_{3}\left(\boldsymbol{x}_{m c}-\boldsymbol{x}_{k-1}^{i}\right)
$$

where $c_{3}$ is the learning factor, $r_{3}$ is the random number, and $\boldsymbol{x}_{m c}$ is the vector of coordinates of the gravity of the swarm.

The vector $\boldsymbol{x}_{m c}$ is given by

$$
\boldsymbol{x}_{m c}=\sum_{i=1}^{N} \boldsymbol{x}^{i} f^{i}(\boldsymbol{x}) / \sum_{i=1}^{N} f^{i}(\boldsymbol{x})
$$

where $f^{i}(\boldsymbol{x})$ is the objective function and $N$ is the number of particles in the swarm.

The effectiveness of the modification of the PSO algorithm was first highlighted in [15]. It was demonstrated that adding a component representing the center of the mass of the swarm helps to improve the convergence of the optimization process of the LSPMSM, thus offers practical benefits, in particular the shortening of computational times.

\section{Case Study}

In order to verify the effectiveness of the proposed strategy, a particular LSPMSM has been considered whose stator comes from a classical $3.5 \mathrm{~kW}$ general purpose induction motor; details of the initial design may be found in [2].

The optimization task has been defined as a search for such a shape of the starting cage rods which-with the given dimensions and distribution of the rotor permanent magnets-would maximize the previously defined parameter $k_{i}$, while maintaining high values of efficiency and power factor. Magnetic field simulation was undertaken using commercial software Maxwell assuming planar symmetry of the machine, supplemented by parameterized shape descriptors of the starting cage, and linked-via appropriate scriptingwith specially developed optimization routines. The algorithm includes two 2-D FEM transient field simulations: 1) a model to calculate efficiency $\eta$ and power factor (PF) under the rated load condition and 2) calculation of the synchronizing torque $T_{80}$ (a torque generated at $80 \%$ of the synchronous speed). As mentioned above, these simulations are carried out in parallel.

The shape of the starting-cage bars has been described by six dimensions defined in Fig. 3. It will be noted that the introduction of the radii $r_{1}$ and $r_{2}$ as design parameters overspecifies the problem and unnecessarily complicates the description of cage geometry; in practice, these radii are selected by considering the cross-sectional area of the rotor tooth at $r=$ const so that $r_{\min }<r \leq r_{\min }+h_{s k}$. Consequently, in the formulated models, a parameter $k_{z}$ has been introduced describing the angular fill of the slot pitch by the rotor bar. Through simple geometrical derivation, with the assumption of rotor teeth sides being parallel under $r_{\text {min }}<r \leq r_{\text {min }}+h_{s k}$, the radii $r_{1}$ and $r_{2}$ may be easily calculated for a given value of $k_{z}$.

As a result of expressing the radii $r_{1}$ and $r_{2}$ in terms of $k_{z}$, the number of components of the vector $z$ of the design parameters has been reduced to $5: z_{1}=s_{o}$ the width of the slot opening, $z_{2}=h_{o}$ the depth of the slot opening, $z_{3}=h_{s k}$ defining the depth of the slot, $z_{4}=r_{p r}$

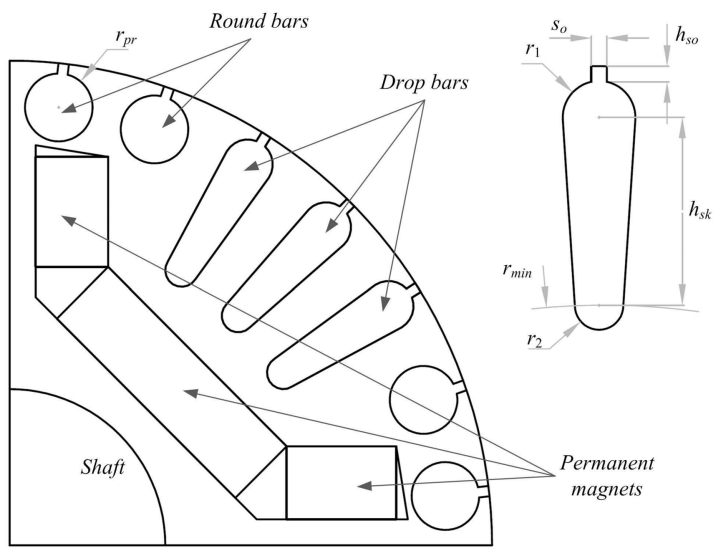

Fig. 3. Design variables describing the shape of the cage bars.

circular bar radius (see Fig. 3), and $z_{5}=k_{z}$ the coefficient introduced above. In the optimization algorithm, the design parameters $z$ describe different quantities with different ranges; in the numerical implementation, these have therefore been normalized [10] and the vector of actual design parameters $z$ replaced by a normalized vector $\boldsymbol{x}$.

The design of permanent magnet machines usually involves multiobjective optimization with multiple parameters and several often conflicting constraints. Here the multiobjective optimization problem has been transformed to a single objective function, combining all objectives, defined as

$$
f(\boldsymbol{x})=\left(\frac{\eta(\boldsymbol{x})}{\eta_{0}}\right)^{q_{1}}\left(\frac{\cos \varphi(\boldsymbol{x})}{\cos \varphi_{0}}\right)^{q_{2}}\left(\frac{T_{80}(\boldsymbol{x})}{T_{0}}\right)^{q_{3}}
$$

where $q_{i}(i=1,2$, and 3$)$ are the weighting coefficients, and $\eta_{0}, \cos \varphi_{0}$, and $T_{0}$ denote the initial values of efficiency, power factor, and the torque $T_{80}$, respectively, assumed in the first iterative step of the optimization process. The weights can be adjusted to suit particular requirements; for the purpose of this paper, they have been assumed to be identical, i.e., $q_{i}=1$.

\section{Results of the Cage Shape Optimization}

Calculations were performed for the following values of the control parameters of the swarm: $N=40, c_{1}=1.2, c_{1}=1.4$, and $c_{3}=1.2$. The number of triangular elements in the 2-D FEM models for synchronous operation and asynchronous operation of the machine were equal to about 35000 and 39000 , respectively. The time step $\Delta t$ for both models was equal to time period/180. The average values of $\eta$, PF, and $T_{80}$ at the start of the iterations were: $92 \%, 0.94$, and $25 \mathrm{Nm}$, respectively. These values were determined by taking an average for several runs of the PSO initiation algorithm.

In order to validate the proposed strategy of evaluation of the synchronizing torque in the optimization of the LSPMSM, field simulations of the starting process were undertaken.

The shapes before and after optimization were considered (Fig. 4). Simulations of the start-up transient were executed for various, gradually increasing, values of the moment of inertia of the load that is for different values of the coefficient $k_{i}$, up to the maximum value $k_{\text {imax }}$, above which the motor fails to synchronize-i.e., rotor speed oscillations do not fade away. Fig. 5 shows the speed waveforms for the 


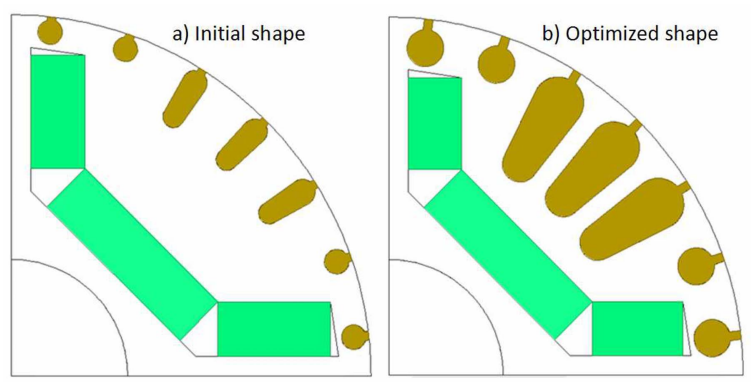

Fig. 4. Initial and optimized shapes of the cage of the LSPMSM. (a) Initial shape. (b) Optimized shape.

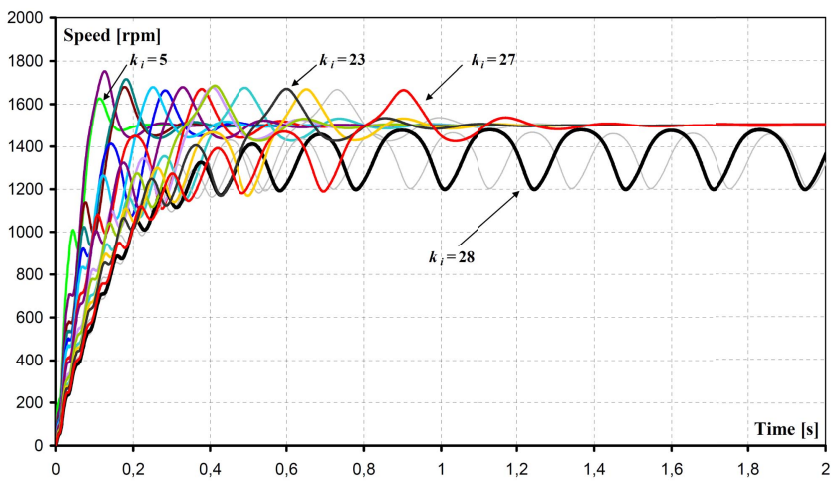

Fig. 5. Speed waveforms during the start-up for the initial shape of the cage.

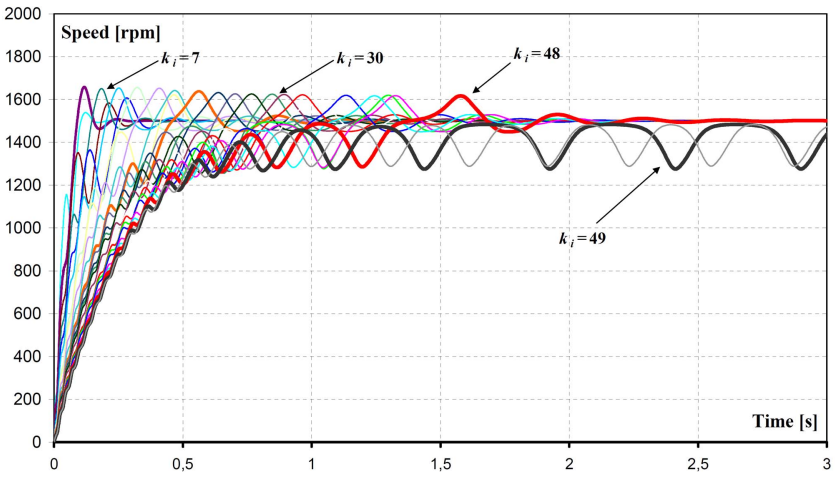

Fig. 6. Speed waveforms during the start-up for the optimized cage.

initial parameters, whereas Fig. 6 contains the results for the optimized machine. It can be seen that the optimized machine can synchronize even for the value of $k_{\text {imax }}=48$, whereas the original motor would only do this for the maximum value of $k_{\text {imax }}=27$.

\section{CONCLUSION}

In this paper, an effective strategy has been put forward for the LSPMSM design optimization. The start-up and operating parameters are established using the numerical field modeling techniques, while the optimization relies on the use of a specially modified PSO approach.

The proposed methodology creates two independent design tracks, both exploiting full field models of the machine. Thus, both the starting and steady-state states are considered, aiming at achieving the best compromise between optimal operating parameters (efficiency and power factor) while delivering the best starting performance $\left(T_{80}\right)$. The simulations are conducted in parallel, which allows the finite-element mesh to be defined to capture the specific properties of the field distributions in both the synchronous and asynchronous states; this improves the accuracy and by itself reduces the computation time by around $15 \%$.

The second innovation is an implementation of the modified PSO algorithm, complemented by dedicated scripting to link the design process with commercial finite-element code, incorporating a specific strategy for assessing the starting performance. This has resulted in a unique integrated dedicated system for design optimization of LSPMSM motors.

The effectiveness of the proposed methodology and computational efficiency of the developed design algorithms were verified by considering a particular case. It was shown that a significant performance improvement was possible; in the case studied, a $75 \%$ increase of the moment of inertia of the load was made possible during starting without sacrificing the steady-state performance.

\section{REFERENCES}

[1] C. Ogbuka, C. Nwosu, and M. Agu, "Dynamic and steady state performance comparison of line-start permanent magnet synchronous motors with interior and surface rotor magnets," Arch. Electr. Eng., vol. 65, no. 1, pp. 105-116, 2016.

[2] Ł. Knypiński, C. Jędryczka, and A. Demenko, "Influence of the shape of squirrel-cage bars on the dimensions of permanent magnets in an optimized line-start permanent magnet synchronous motor," COMPELInt. J. Comput. Math. Elect. Electron. Eng., vol. 36, no. 1, pp. 298-308, 2017.

[3] Ł. Knypiński, L. Nowak, and A. Demenko, "Optimization of the synchronous motor with hybrid permanent magnet excitation system," COMPEL-Int. J. Comput. Math. Elect. Electron. Eng., vol. 34, no. 2, pp. 448-455, 2015.

[4] C. Jedryczka, R. Wojciechowski, and A. Demenko, "Influence of squirrel cage geometry on the synchronisation of the line start permanent magnet synchronous motor," IET Sci. Meas. Techn., vol. 9, no. 2, pp. 197-203, 2015.

[5] M. Barański and C. Jędryczka, "Analysis of the line start permanent magnet synchronous motor performance under different values of the supply voltage," in Proc. Int. Symp. Electromagn. Phenomena Nonlinear Circuits (EPNC), Pilsen, Czech Republic, Jul. 2014, pp. 27-28.

[6] A. Belahcen, F. Martin, M. E.-H. Zaim, E. Dlala, and Z. Kolondzovski, "Combined FE and particle swarm algorithm for optimization of high speed PM synchronous machine," COMPEL-Int. J. Comput. Math. Elect. Electron. Eng., vol. 34, no. 2, pp. 475-484, 2015.

[7] F. Moussouni, S. Brisset, and P. Brochet, "Comparison of two multiagent algorithms: ACO and PSO for the optimization of a brushless DC wheel motor," in Intelligent Computer Techniques in Applied Electromagnetics. Berlin, Germany: Springer-Verlag, 2008, pp. 3-10.

[8] D. Haibin, S. Li, and Y. Shi, "Predator-prey brain storm optimization for DC brushless motor," IEEE Trans. Magn., vol. 49, no. 10, pp. 5336-5340, Oct. 2013.

[9] T. C. Bora, L. dos S. Coelho, and L. Lebensztajn, "Bat-inspired optimization approach for the brushless DC wheel motor problem," IEEE Trans. Magn., vol. 48, no. 2, pp. 947-950, Feb. 2012.

[10] Y. L. Karnavas, I. D. Chasiotis, and E. L. Peponakis, "Permanent magnet synchronous motor design using grey wolf optimizer algorithm," Int. J. Electr. Comput. Eng., vol. 40, no. 3, pp. 1353-1362, 2016.

[11] J. Kennedy and R. Eberhart, "Particle swarm optimization," in Proc. Int. Conf. Neutral Netw., Perth, WA, Australia, 1995, pp. 1942-1948.

[12] S. Kiranyaz, T. Ince, A. Yildirim, and M. Gabbouj, "Fractional particle swarm optimization in multidimensional search space," IEEE Trans. Syst., Man, Cybern. B, Cybern., vol. 40, no. 2, pp. 298-319, Apr. 2010.

[13] Z. D. Zaharis and T. V. Yioultsis, "A novel adaptive beamforming technique applied on linear antenna arrays using adaptive mutated Boolean PSO," Progr. Electromagn. Res., vol. 117, pp. 165-179, Aug. 2011.

[14] A.-Q. Mu, D.-X. Cao, and X.-H. Wang, "A modified particle swarm optimization algorithm," Natural Sci., vol. 1, no. 2, pp. 151-155, 2009.

[15] Ł. Knypiński, L. Nowak, and C. Jędryczka, "Optimization of the rotor geometry of the line-start permanent magnet synchronous motor by the use of particle swarm optimization," COMPEL-Int. J. Comput. Math. Elect. Electron. Eng., vol. 34, no. 3, pp. 882-892, 2015. 